Ilchmann, Achim; Isidori, A. :

Adaptive dynamic output feedback stabilisation of nonlinear systems

Zuerst erschienen in:

Asian Journal of Control 4 (2002), S. 1-9 


\title{
Adaptive dynamic output feedback stabilisation of nonlinear systems
}

\author{
A. Ilchmann* and A. Isidori ${ }^{\dagger}$
}

August 1, 2001

\begin{abstract}
An adaptive dynamical output feedback controller is introduced for a class of nonlinear non-minimum phase systems. This adaptive controller achieves practical stabilisation, that means the output will asymptotically tend to a pre-specified neighbourhood of the origin. In case of linear systems, we can even prove adaptive stabilisation.
\end{abstract}

Keywords: adaptive control, dynamic output feedback, nonlinear systems, practical adaptive stabilisation

\section{Nomenclature}

$$
\begin{aligned}
& \mathcal{L}^{p}\left(I ; \mathbb{K}^{n \times m}\right) \quad \text { set of } p \text {-integrable functions } f: I \rightarrow \mathbb{K}^{n \times m}, I \subset \mathbb{R} \text { an interval, } \\
& p \geq 1 \\
& \|f\|_{\mathcal{L}^{p}\left(I ; \mathbb{K}^{n \times m}\right)}=\left(\int_{I}\|f(\tau)\|^{p} d \tau\right)^{1 / p} \\
& \mathcal{L}^{\infty}\left(I ; \mathbb{K}^{n \times m}\right) \quad \text { set of functions } f: I \rightarrow \mathbb{K}^{n \times m} \text { which are essentially bounded on } \\
& \text { the interval } I \subset \mathbb{R} \\
& \|f\|_{\mathcal{L}^{\infty}\left(I ; \mathbb{K}^{n \times m}\right)}=\operatorname{ess} \sup _{t \in I}\|f(t)\|
\end{aligned}
$$

\section{Introduction}

In this paper, we consider polynomially bounded nonlinear systems of the form

$$
\dot{z}(t)=f(z(t), y(t)), \quad \dot{y}(t)=q(z(t), y(t))+b(y(t)) u(t),
$$

where it is assumed that $f: \mathbb{R}^{n-1} \times \mathbb{R}^{m} \longrightarrow \mathbb{R}^{n-1}, q: \mathbb{R}^{n-1} \times \mathbb{R}^{m} \longrightarrow \mathbb{R}^{m}, b: \mathbb{R}^{m} \longrightarrow$ $\mathbb{R}^{m \times m}$ are locally Lipschitz, with $f(0,0)=0, q(0,0)=0, b(y)$ is invertible for all $y \in \mathbb{R}^{m}$, and there exist real numbers $\mu_{f}, \mu_{q}, \mu_{q 0}>0$ and an integer $s \geq 1$ such that, for all $z \in \mathbb{R}^{(n-1)}, \tilde{y}, y \in \mathbb{R}^{m}$, we have

$$
\begin{aligned}
\|f(z, \tilde{y})-f(z, y)\| & \leq \mu_{f}\left[1+\|\tilde{y}-y\|^{s}\right] \\
\|q(z, \tilde{y})-q(z, y)\| & \leq \mu_{q}\left[1+\|\tilde{y}-y\|^{s}\right] \\
\|q(z, 0)\| & \leq \mu_{q 0}\left[1+\|z\|^{s}\right] .
\end{aligned}
$$

${ }^{*}$ Institut für Mathematik, Technische Universität Ilmenau, Weimarer Straße 25, 98693 Ilmenau, ilchmann@mathematik.tu-ilmenau.de

${ }^{\dagger}$ Dipartimento di Infomatica e Sistemistica, Università di Roma "La Sapienza", 00184 Rome, Italy, isidori@labrob.ing. uniroma1.it 
The control problem that we address is the design of a dynamic (and adaptive) output feedback law yielding global practical stability. More precisely, we seek a family of dynamical systems of the form

$$
\dot{\hat{x}}=\chi_{\varepsilon}(\hat{x}, y), \quad u=p_{\varepsilon}(\hat{x}, y)
$$

where $\lambda>0$ is a constant design parameter, such that, in the resulting closed-loop system $(1.1,1.2)$, for every initial condition $z(0), y(0), \hat{x}(0)$

- the solution $(z(t), y(t), \hat{x}(t))$ exists for all $t \in[0, \infty)$ and is bounded,

- $\lim _{t \rightarrow \infty} \max \{0,\|y(t)\|-\lambda\}=0$, i.e. the distance between $\|y(t)\|$ and $[0, \lambda]$ tends to zero as $t \rightarrow \infty$.

The family of controllers that we design is such that, in the linear case we can choose $\lambda=0$ and $z(t)$ and $y(t)$. In the non-linear case, for any $\lambda>0$, there is a controller in the family (1.2) such that $\|z(t)\|$ asymptotically converges to the strip $[0, \lambda]$ as time tends to $\infty$.

The basic assumption that we make on system (1.1) in order to meet this control objective is that the following auxiliary system associated with (1.1)

$$
\dot{z}(t)=f(z(t), \bar{u}(t)), \quad \bar{y}(t)=q(z(t), \bar{u}(t)),
$$

regarded as a system with input $\bar{u}$, output $\bar{y}$ and internal state $z$, is globally asymptotically stabilisable by a dynamic output feedback of the form

$$
\dot{\eta}(t)=L(\eta(t))+M \bar{y}(t), \quad \bar{u}(t)=N(\eta(t)),
$$

in which $M \in \mathbb{R}^{q \times m}$ and $L: \mathbb{R}^{q} \longrightarrow \mathbb{R}^{q}$ is locally Lipschitz, with $L(0)=0, N: \mathbb{R}^{q} \longrightarrow \mathbb{R}^{m}$ is continuously differentiable and there exists real numbers $\mu_{L}, \mu_{N}>0$ and an integer $r \geq 1$ such that, for all $\eta, \tilde{\eta} \in \mathbb{R}^{q}$, we have

$$
\begin{aligned}
\|L(\tilde{\eta})-L(\eta)\| & \leq \mu_{L}\left[1+\|\tilde{\eta}-\eta\|^{s}\right] \\
\|N(\tilde{\eta})-N(\eta)\| & \leq \mu_{N}\left[1+\|\tilde{\eta}-\eta\|^{r}\right] .
\end{aligned}
$$

More precisely it is assumed that (1.5) renders the equilibrium $(z, \eta)=(0,0)$ of the closedloop system

$$
\begin{aligned}
& \dot{z}(t)=f(z(t), N(\eta(t))) \\
& \dot{\eta}(t)=L(\eta(t))+M q(z(t), N(\eta(t)))
\end{aligned}
$$

globally asymptotically stable, and there exist a continuously differentiable Lyapunov function $W: \mathbb{R}^{n-1} \times \mathbb{R}^{q} \rightarrow \mathbb{R}_{\geq 0}$ and real numbers $w_{1}, w_{2}, w_{3}, w_{4}$ such that, for all $\xi=$ $\left(z^{T}, \eta^{T}\right)^{T} \in \mathbb{R}^{n-1+q}$,

$$
\begin{aligned}
w_{1}\|\xi\| \leq W(z, \eta) & \leq w_{2}\|\xi\| \\
\operatorname{grad} W(z, \eta) \cdot\left[\begin{array}{c}
f(z, N(\eta)) \\
L(\eta)+M(q(z), N(\eta))
\end{array}\right] & \leq-w_{3}\|\xi\| \\
\|\operatorname{grad} W(z, \eta)\| & \leq w_{4} .
\end{aligned}
$$


This type of assumption was proven in [5] to be helpful in determining a (dynamic) output feedback law that semiglobally practically stabilises a nonlinear system of the form (1.1). In the present paper we extend the result of [5] by showing that, if a certain "gain parameter" included in the feedback law is continuously adapted (rather than fixed as in [5]), then the feedback law steers the state of (1.1) to an arbitrarily small neighborhood of the origin regardless of the initial value (and not just for any initial value in a fixed, possibly large, compact set as in [5]). The adaptation law used in the present paper is simple. It is a time-varying gain driven by an integration in the linear case, and in the nonlinear case an integration coupled with a dead-zone. This high-gain idea goes back to [10] and, if a dead-zone is incorporated, to [4], see also [2] for polynomially bounded systems. It is worth observing that (as noted in [5]), in the case of linear systems, the assumption in question (see (1.4), (1.5)) is not restrictive at all: it is fulfilled by any system which is stabilisable by means of dynamic output feedback. We also observe (as pointed out in [6]) that the assumption in question essentially identifies a class of nonlinear systems that are semiglobally stabilisable by means of a feedback driven by functions that are "uniformly completely observable" in the sense of [9].

\section{Linear case}

In this section, we consider the special case in which system (1.1) is a linear system, namely a system of the form

$$
\begin{aligned}
& \dot{z}(t)=F z(t)+G y(t) \\
& \dot{y}(t)=H z(t)+J y(t)+B u(t),
\end{aligned}
$$

where $F \in \mathbb{R}^{(n-1) \times(n-1)}, G \in \mathbb{R}^{(n-1) \times m}, H \in \mathbb{R}^{m \times(n-1)}, J \in \mathbb{R}^{m \times m}$, and $B \in \mathbb{R}^{m \times m}$ is invertible.

The corresponding version of the basic assumption introduced in the previous section (see also Figure 1) is that the following subsystem of (2.1)

$$
\begin{aligned}
& \dot{z}(t)=F z(t)+G \bar{u}(t) \\
& \bar{y}(t)=H z(t)+J \bar{u}(t),
\end{aligned}
$$

is uniformly asymptotically stabilisable by a dynamic output feedback of the form

$$
\dot{\eta}(t)=L \eta(t)+M \bar{y}(t), \quad \bar{u}(t)=N \eta(t),
$$

where $L \in \mathbb{R}^{q \times q}, M \in \mathbb{R}^{q \times m}, N \in \mathbb{R}^{m \times q}$.

The following Proposition 2.1 is an adaptive version of Lemma 3.1 in [5], where it was assumed that, in the feedback law $u(\cdot), k(\cdot) \equiv k^{*}$ is sufficiently large.

Proposition 2.1 Suppose (2.3) applied to (2.2) yields a uniformly asymptotically stable closed-loop system. Then the dynamic and adaptive output feedback controller

$$
\begin{aligned}
\dot{\eta}(t) & =L \eta(t)+M k(t)[y(t)-N \eta(t)]) \\
u(t) & =B^{-1}[N[L \eta+M k(t)[y(t)-N \eta(t)]]-k(t)[y(t)-N \eta(t)]] \\
\dot{k}(t) & =\|y(t)-N \eta(t)\|^{r}
\end{aligned}
$$




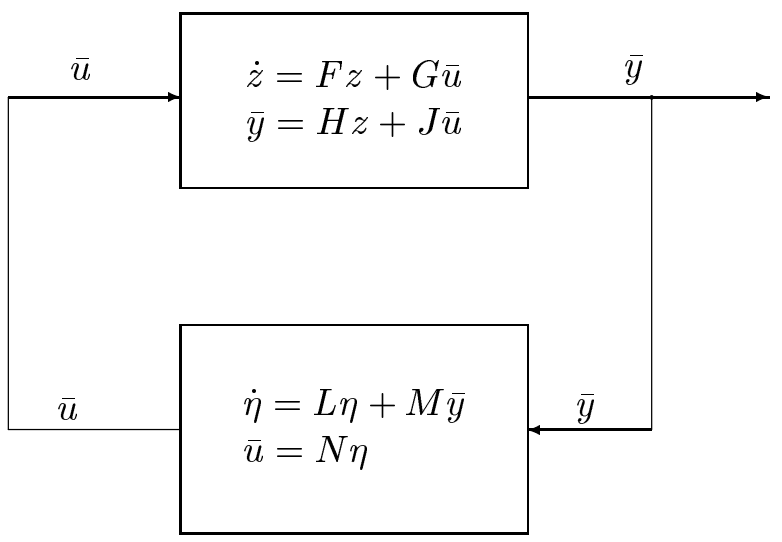

Figure 1: Assumption on global asymptotic stabilisability: Stable closed-loop system

with $r \geq 1$, applied to (2.1) yields, for arbitrary initial data $(z(0), y(0), \eta(0), k(0)) \in$ $\mathbb{R}^{n-1} \times \mathbb{R}^{m} \times \mathbb{R}^{q} \times \mathbb{R}$, a nonlinear closed-loop system (2.1), (2.4) which possesses a unique absolutely continuous solution

$$
(z(\cdot), y(\cdot), \eta(\cdot), k(\cdot)):[0, \omega) \longrightarrow \quad \mathbb{R}^{n-1} \times \mathbb{R}^{m} \times \mathbb{R}^{q} \times \mathbb{R},
$$

maximally extended over $[0, \omega)$, where $\omega \in(0, \infty]$, and has the properties

(i) $\omega=\infty$, i.e. no finite escape time,

(ii) $\lim _{t \rightarrow \infty} k(t)=k_{\infty} \in \mathbb{R}_{\geq 0}$, i.e. the gain adaptation converges,

(iii) $z(\cdot), y(\cdot), \eta(\cdot) \in \mathcal{L}^{r}\left(\mathbb{R}_{\geq 0} ; \mathbb{R}^{n-1}\right) \times \mathcal{L}^{r}\left(\mathbb{R}_{\geq 0} ; \mathbb{R}^{m}\right) \times \mathcal{L}^{r}\left(\mathbb{R}_{\geq 0} ; \mathbb{R}^{q}\right)$, and $\lim _{t \rightarrow \infty}\|z(t)\|+\|y(t)\|+\|\eta(t)\|=0, \quad$ i.e. stabilisation.

The proof of Proposition 2.1 and of the subsequent results relies on the following lemma. Although the result might be well-known, we are not aware of a concise reference, and present a short proof.

Lemma 2.2 Consider the differential equation

$$
\dot{x}(t)=F(t, x(t))+\varphi_{1}(t)+\varphi_{2}(t),
$$

where $F(\cdot, \cdot): \mathbb{R}_{\geq 0} \times \mathbb{R}^{n} \rightarrow \mathbb{R}^{n}, \varphi_{i}(\cdot): \mathbb{R}_{\geq 0} \rightarrow \mathbb{R}^{n}, i=1,2$, and suppose there exists a continuously differentiable Lyapunov function $V(\cdot, \cdot): \mathbb{R}_{\geq 0} \times \mathbb{R}^{n} \rightarrow \mathbb{R}_{\geq 0}$ so that, for some $\alpha_{1}, \ldots, \alpha_{4}>0, p \geq 1$, and all $(t, x) \in \mathbb{R}_{\geq 0} \times \mathbb{R}^{n}$,

$$
\begin{aligned}
\alpha_{1}\|x\|^{p} \leq V(t, x) & \leq \alpha_{2}\|x\|^{p} \\
\frac{\partial}{\partial t} V(t, x)+\frac{\partial}{\partial x} V(t, x) \cdot F(t, x) & \leq-\alpha_{3}\|x\|^{p} \\
\|\operatorname{grad} V(t, x)\| & \leq \alpha_{4} .
\end{aligned}
$$

Then any absolutely continuous solution $x(\cdot): \mathbb{R}_{\geq 0} \rightarrow \mathbb{R}^{n}$ of (2.5) satisfies, for any $r \geq 1$ :

(i) $\varphi_{1}(\cdot) \in L^{r}\left(\mathbb{R}_{\geq 0} ; \mathbb{R}^{n}\right) \wedge \lim _{t \rightarrow \infty} \varphi_{2}(t)=0$

$\Longrightarrow \quad \lim _{t \rightarrow \infty} x(t)=0$,

(ii) $\varphi_{1}(\cdot) \in L^{r}\left(\mathbb{R}_{\geq 0} ; \mathbb{R}^{n}\right) \wedge \varphi_{2}(\cdot) \in L^{\infty}\left(\mathbb{R}_{\geq 0} ; \mathbb{R}^{n}\right) \quad \Longrightarrow \quad x(\cdot) \in L^{\infty}\left(\mathbb{R}_{\geq 0} ; \mathbb{R}^{n}\right)$. 


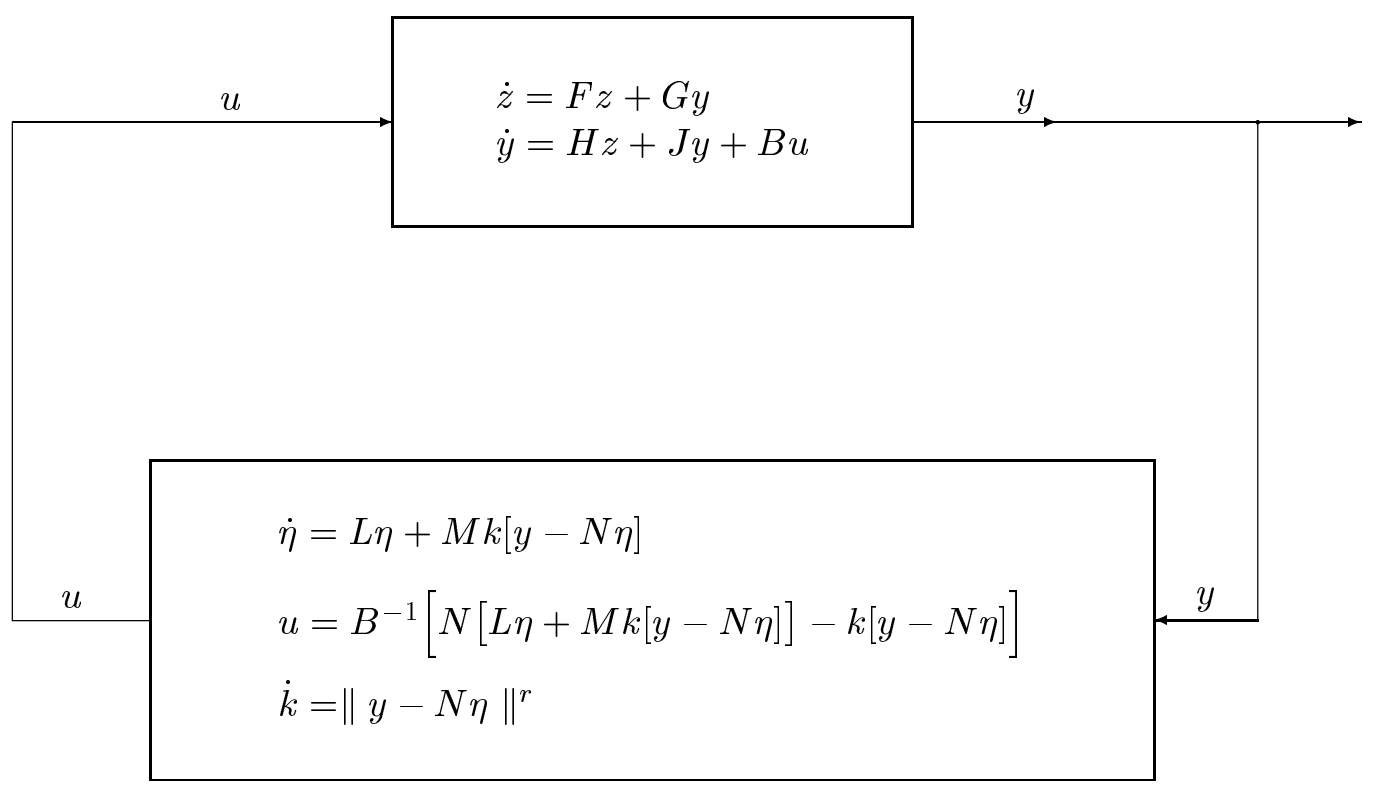

Figure 2: Linear case: Global stabilisation by dynamic output feedback and gain adaption

Proof: The derivative of $V(t, x(t))$ along a solution of (2.5) satisfies, for almost all $t \geq 0$,

$$
\frac{\mathrm{d}}{\mathrm{d} t} V(t, x(t)) \leq-\frac{\alpha_{3}}{\alpha_{2}} V(t, x(t))+\alpha_{4}\left[\left\|\varphi_{1}(t)\right\|+\left\|\varphi_{2}(t)\right\|\right],
$$

and hence, applying Variations-of-Constants yields, for all $t \geq t_{0} \geq 0$,

$$
V(t, x(t)) \leq e^{-\frac{\alpha_{3}}{\alpha_{2}}\left(t-t_{0}\right)} V\left(t_{0}, x\left(t_{0}\right)\right)+\int_{t_{0}}^{t} e^{-\frac{\alpha_{3}}{\alpha_{2}}(t-s)} \alpha_{4}\left[\left\|\varphi_{1}(s)\right\|+\left\|\varphi_{2}(s)\right\|\right] d s .
$$

Note that by Hölder's inequality we have, for $1 / p+1 / q=1$ and all $t \geq t_{0} \geq 0$,

$$
\begin{aligned}
& \int_{t_{0}}^{t} e^{-\alpha(t-s)}\|\varphi(s)\| d s \\
& \leq\left\|e^{-\alpha \cdot}\right\|_{L^{p}\left(\frac{t-t_{0}}{2}, t-t_{0}\right)}\|\varphi(\cdot)\|_{L^{q}\left(t_{0}, \frac{t+t_{0}}{2}\right)}+\left\|e^{-\alpha \cdot}\right\|_{L^{p}\left(0, \frac{t-t_{0}}{2}\right)}\|\varphi(\cdot)\|_{L^{q}\left(\frac{t+t_{0}}{2}, t\right)} \\
& \leq\left\|e^{-\alpha \cdot}\right\|_{L^{p}\left(\frac{t-t_{0}}{2}, \infty\right)}\|\varphi(\cdot)\|_{L^{q}\left(t_{0}, \infty\right)}+\left\|e^{-\alpha \cdot}\right\|_{L^{p}(0, \infty)}\|\varphi(\cdot)\|_{L^{q}\left(\frac{t+t_{0}}{2}, \infty\right)}
\end{aligned}
$$

The statements of the lemma follow from applying (2.8) to the convolution in (2.7) and invoking (2.6) again.

Proof of Proposition 2.1: It follows from standard results of ordinary differential equations that the closed-loop system $(2.1),(2.4)$ possesses a unique absolutely continuous solution which can be maximally extended over an interval $[0, \omega)$, where $\omega \in(0, \infty]$. 
The $(z, y, \eta)$-coordinates of the closed-loop system satisfy, where for brevity we omit the argument $t$,

$$
\begin{array}{ll}
\dot{z}=F z+G N \eta & +G[y-N \eta] \\
\dot{y}=H z+J y & +N L \eta
\end{array}
$$

and the coordinate transformation $\theta:=y-N \eta$ leads to

$$
\begin{aligned}
& \dot{z}=F z+G[N \eta+\theta] \\
& \dot{\eta}=L \eta+M k \theta \\
& \dot{\theta}=H z+J[\theta+N \eta]-k \theta
\end{aligned}
$$

so that the further coordinate transformation $\zeta:=\eta+M \theta$ yields, for $\xi=\left(z^{T}, \zeta^{T}\right)^{T}$,

$$
\begin{aligned}
& \dot{\xi}=\hat{A} \xi+\hat{B} \theta \\
& \dot{\theta}=\hat{C} \xi-\left[k(t) I_{m}-\hat{D}\right] \theta,
\end{aligned}
$$

where

$$
\begin{aligned}
& \hat{A}=\left[\begin{array}{cc}
F, & G N \\
M H, & {[L+M J N]}
\end{array}\right], \quad \hat{B}=\left[\begin{array}{c}
G\left[I_{m}-N M\right] \\
M J-[L+M J N] M
\end{array}\right], \\
& \hat{C}=[H, J N], \quad \hat{D}=[J-J N M] .
\end{aligned}
$$

Note that $\hat{A}$ is the matrix of the closed-loop system (2.2), (2.3), and hence by assumption exponentially stable.

We prove that $k(\cdot) \in \mathcal{L}^{\infty}(0, \omega ; \mathbb{R})$. Seeking a contradiction, suppose $k(\cdot)$ is unbounded. Let $P=P^{T} \in \mathbb{R}^{(n-1+q) \times(n-1+q)}$ be the positive definite solution of

$$
\hat{A}^{T} P+P \hat{A}=-I \text {. }
$$

Then differentiation of the Lyapunov function candidate

$$
V(\xi, \theta)=\xi^{T} P \xi+\frac{1}{2}\|\theta\|^{2}, \quad \text { where } \quad \xi=\left(z^{T}, \zeta^{T}\right)^{T}
$$

along the solution of (2.11) yields, for almost all $t \in[0, \omega)$,

$$
\begin{aligned}
\frac{\mathrm{d}}{\mathrm{d} t} V(\xi(t), \theta(t)) & =-\|\xi(t)\|^{2}+2 \xi(t)^{T} P \hat{B} \theta(t)-\theta(t)^{T}\left[k(t) I_{m}-\hat{D}\right] \theta(t)+\theta(t)^{T} \hat{C} \xi(t) \\
& \leq-\|\xi(t)\|^{2}-[k(t)-\|\hat{D}\|]\|\theta(t)\|^{2}+[2\|P \hat{B}\|+\|\hat{C}\|]\|\xi(t)\|\|\theta(t)\| \\
& \leq-\frac{1}{2}\|\xi(t)\|^{2}-\left[k(t)-\|\hat{D}\|-2[2\|P \hat{B}\|+\|\hat{C}\|]^{2}\right]\|\theta(t)\|^{2} .
\end{aligned}
$$

Now monotonicity and unboundedness of $k(\cdot)$ yields exponential decay of $(z(t), \zeta(t), \theta(t))$ on $[0, \omega)$, and by the coordinate transformations we may conclude exponential decay of $(z(t), y(t), \eta(t))$ on $[0, \omega)$. Thus $t \mapsto k(t)$ converges as $t$ tends to $\omega$, which contradicts unboundedness of $k(\cdot)$.

$k(\cdot) \in \mathcal{L}^{\infty}(0, \omega ; \mathbb{R})$ readily gives $\omega=\infty$, and hence (i) and (ii) are proved and it remains to show (iii).

$k(\cdot) \in \mathcal{L}^{\infty}(0, \infty ; \mathbb{R})$ is equivalent to $\theta(\cdot) \in \mathcal{L}^{r}\left(\mathbb{R}_{\geq 0} ; \mathbb{R}^{m}\right)$ and hence an application of Lemma 2.2 to $(2.11)$ yields $(z(\cdot), \zeta(\cdot)) \in \mathcal{L}^{r}\left(\mathbb{R}_{\geq 0} ; \mathbb{R}^{n-1} \times \mathbb{R}^{q}\right)$ and $\lim _{t \rightarrow \infty}(z(t), \zeta(t))=0$. 
This gives $\eta(\cdot)=\zeta(\cdot)-M \theta(\cdot) \in \mathcal{L}^{r}\left(\mathbb{R}_{\geq 0} ; \mathbb{R}^{q}\right)$ and $y(\cdot)=\theta(\cdot)+N \eta(\cdot) \in \mathcal{L}^{r}\left(\mathbb{R}_{\geq 0} ; \mathbb{R}^{m}\right)$, and a repeated application of Lemma 2.2 to

$$
\dot{y}(t)=-y(t)+\mu(z(t), y(t), \eta(t)), \quad \text { where } \quad \mu(z, y, \eta):=y+H z+J y+B u,
$$

gives $\lim _{t \rightarrow \infty} y(t)=0$. Finally, rewriting the lower equation in (2.9) as

$$
\dot{\eta}=-\eta+[I+L-M k N] \eta+M k y,
$$

gives, again by Lemma $2.2, \lim _{t \rightarrow \infty} \eta(t)=0$. This completes the proof of the theorem.

It is easy to see that the gain adaptation law can be replaced by $\dot{k}=\|y\|^{r}+\|N \eta\|^{r}$ or $\dot{k}=\|y\|^{r}+\|\eta\|^{r}$.

\section{Nonlinear case}

The following theorem generalises Proposition 2.1. However, stabilisation to zero can no longer be guaranteed and is replaced by the weaker control objective of practical output stabilisation. The following theorem also generalizes Theorem 4.1 in [5], where practical stabilisation with arbitrary large basin of convergence is ensured for $k(\cdot) \equiv k^{*}$ sufficiently large. The basic difference in the control law (3.1) is that the gain parameter $k(t)$ is tuned adaptively, making it possible to obtain the desired convergence properties for any initial data.

Theorem 3.1 Suppose (1.4) applied to (1.5) yields a globally uniformly asymptotically stable closed-loop system in the sense that there exists some $W: \mathbb{R}^{n-1} \times \mathbb{R}^{q} \rightarrow \mathbb{R}_{\geq 0}$ satisfying (1.5). Let $r_{1} \geq 1, \lambda_{1}, \lambda_{2}>0$ be given, and $r, s \geq 1$ as in (1.2), (1.6). Then the dynamic and adaptive output error feedback controller

$$
\begin{aligned}
\theta(t) & =y(t)-N(\eta(t)) \\
\dot{\eta}(t) & =L(\eta(t))+M k(t)\|\theta(t)\|^{r s-1} \theta(t), \\
u(t) & =b(y(t))^{-1}\left[\frac{\partial N}{\partial \eta}(\eta(t))\left[L(\eta(t))+M k(t)\|\theta(t)\|^{r s-1} \theta(t)\right]-k(t)\|\theta(t)\|^{r s-1} \theta(t)\right] \\
\dot{k}(t) & =\max \left\{0,\|y(t)\|-\lambda_{1}\right\}^{r_{1}}+\max \left\{0,\|\theta(t)\|-\lambda_{2}\right\}^{r s},
\end{aligned}
$$

applied to (1.1) yields, for arbitrary initial data $(z(0), y(0), \eta(0), k(0)) \in \mathbb{R}^{n-1} \times \mathbb{R}^{m} \times$ $\mathbb{R}^{q} \times \mathbb{R}$, a closed-loop system (1.1), (3.1) which possesses a unique absolutely continuous solution

$$
(z(\cdot), y(\cdot), \eta(\cdot), k(\cdot)):[0, \omega) \quad \longrightarrow \quad \mathbb{R}^{n-1} \times \mathbb{R}^{m} \times \mathbb{R}^{q} \times \mathbb{R}
$$

maximally extended over $[0, \omega)$, where $\omega \in(0, \infty]$, and has the properties

(i) $\omega=\infty$, i.e. no finite escape time,

(ii) $\lim _{t \rightarrow \infty} k(t)=k_{\infty} \in \mathbb{R}_{\geq 0}$, i.e. the gain adaptation converges,

(iii) $z(\cdot), y(\cdot), \eta(\cdot) \in \mathcal{L}^{\infty}\left(\mathbb{R}_{\geq 0} ; \mathbb{R}^{n-1}\right) \times \mathcal{L}^{\infty}\left(\mathbb{R}_{\geq 0} ; \mathbb{R}^{m}\right) \times \mathcal{L}^{\infty}\left(\mathbb{R}_{\geq 0} ; \mathbb{R}^{q}\right)$,

i.e. all signals are bounded, 
(iv) $\lim _{t \rightarrow \infty} \max \left\{0,\|y(t)\|-\lambda_{1}\right\}=0, \quad$ and $\quad \lim _{t \rightarrow \infty} \max \left\{0,\|\theta(t)\|-\lambda_{2}\right\}=0$, i.e. $\|y(t)\|$ and $\mid \theta(t) \|$ approach $\left[0, \lambda_{1}\right]$ and $\left[0, \lambda_{2}\right]$ respectively as $t$ tends to $\infty$.

Moreover, for any $\varepsilon>0$ there is $\lambda_{2}^{*}$ such that, if $\lambda_{2} \leq \lambda_{2}^{*}$, then

(v) $\lim _{t \rightarrow \infty} \max \{0,\|z(t)\|+\|\eta(t)\|-\varepsilon\}=0$.

Note the essential difference between statements (iv) and (v) in Theorem 3.1: For prespecified and arbitrary small $\lambda_{1}, \lambda_{2}>0$ it is guaranteed that eventually $y(t)$ and $\theta(t)$ stay in a $\lambda_{1}$ - respectively $\lambda_{2}$-neighbourhood of 0 . This cannot be guaranteed a priori for the $z(t)$ and $\eta(t)$-components. Statement $(\mathrm{v})$ only guarantees qualitatively that the smaller eventually $\theta(t)$ is the smaller the other internal states $z(t)$ and $\eta(t)$ eventually become.

Proof of Theorem 3.1: The $(z, y, \eta)$-coordinates of the closed-loop system (1.1),(3.1) may be written as

$$
\begin{aligned}
& \dot{z}=f(z, \theta+N(\eta)) \\
& \dot{y}=q(z, \theta+N(\eta))+\frac{\mathrm{d}}{\mathrm{d} t} N(\eta)-k\|\theta\|^{r s-1} \theta \\
& \dot{\eta}=L(\eta) \quad+M k\|\theta\|^{r s-1} \theta \text {, }
\end{aligned}
$$

and hence it follows from standard results of ordinary differential equations that the closedloop system possesses a unique absolutely continuous solution which can be maximally extended over an interval $[0, \omega)$, where $\omega \in(0, \infty]$.

The coordinate transformation $\zeta:=\eta+M \theta$ yields

$$
\begin{aligned}
\dot{z} & =f(z, \theta+N(\zeta-M \theta)) \\
\dot{\zeta} & =L(\zeta-M \theta)+M q(z, \theta+N(\zeta-M \theta)) \\
\dot{\theta} & =-k\|\theta\|^{r s-1} \theta+q(z, \theta+N(\zeta-M \theta)) .
\end{aligned}
$$

In order to make use of the global uniform asymptotic stability of (1.7), we rewrite (3.3) as follows.

$$
\begin{array}{ll}
\dot{z}=f(z, N(\zeta)) & +\delta_{z}(z, \zeta, \theta) \\
\dot{\zeta}=L(\zeta)+M q(z, N(\zeta)) & +\delta_{\zeta}(z, \zeta, \theta) \\
\dot{\theta}=-k\|\theta\|^{r s-1} \theta & +q(z, \theta+N(\zeta-M \theta)),
\end{array}
$$

where

$$
\begin{aligned}
& \delta_{z}(z, \zeta, \theta)=f(z, \theta+N(\zeta-M \theta))-f(z, N(\zeta)) \\
& \delta_{\zeta}(z, \zeta, \theta)=L(\zeta-M \theta)-L(\zeta)+M[q(z, \theta+N(\zeta-M \theta))-q(z, N(\zeta))] .
\end{aligned}
$$

For the sake of simplicity we redefine the distance function, for $\lambda>0$ and $\theta \in \mathbb{R}^{p}, p$ being given by the context,

$$
d_{\lambda}(\theta)=\max \{0,\|\theta\|-\lambda\} .
$$

Using the following inequality (see, e.g. [8, Section XI.4]),

$$
[a+b]^{k} \leq 2^{k-1}\left[a^{k}+b^{k}\right] \quad \text { for all } a, b \geq 0 \text { and } k \in \mathbb{N},
$$


and (1.2), (1.6), (1.8), we conclude that the derivative of $W(z(t), \zeta(t))$ along the solution of (3.3) satisfies, for $\tilde{w}_{3}:=w_{3} / w_{2}$ and suitable constants $\mu_{1}, \mu_{2}>0$ and almost all $t \in[0, \omega)$,

$$
\begin{aligned}
\frac{\mathrm{d}}{\mathrm{d} t} W(z(t), \zeta(t))= & \operatorname{grad} W(z, \zeta) \cdot \frac{\mathrm{d}}{\mathrm{d} t}\left(z^{T}, \zeta^{T}\right)^{T} \\
\leq & -w_{3}\left\|\left(z^{T}, \zeta^{T}\right)^{T}\right\|+w_{4}\left\|\left(\delta_{z}^{T}, \delta_{\zeta}^{T}\right)^{T}\right\| \\
\leq & -w_{3}\left\|\left(z^{T}, \zeta^{T}\right)^{T}\right\|+w_{4}\left\{\mu_{f}\left[1+\|\theta+N(\zeta-M \theta)-N(\zeta)\|^{s}\right]\right. \\
& \left.+\mu_{L}\left[1+\|M \theta\|^{s}\right]+\|M\| \mu_{q}\left[1+\|\theta+N(\zeta-M \theta)-N(\zeta)\|^{s}\right]\right\} \\
\leq & -w_{3}\left\|\left(z^{T}, \zeta^{T}\right)^{T}\right\|+\mu_{1}\left[1+\|\theta\|^{s}+\|N(\zeta-M \theta)-N(\zeta)\|^{s}\right] \\
\leq & -w_{3}\left\|\left(z^{T}, \zeta^{T}\right)^{T}\right\|+\mu_{2}\left[1+\|\theta\|^{r s}\right] \\
\leq & -\tilde{w}_{3} W(z, \zeta)+\mu_{2}\left[1+\|\theta\|^{r s}\right] .
\end{aligned}
$$

Since (3.8) is equivalent to

$$
\frac{\mathrm{d}}{\mathrm{d} t}\left[e^{\tilde{w}_{3} t} W(z(t), \zeta(t))\right] \leq e^{\tilde{w}_{3} t} \mu_{2}\left[1+\|\theta(t)\|^{r s}\right],
$$

it follows from (1.8), the decomposition of $\|\theta\|^{\text {rs }}$ into $d_{\lambda}(\theta)^{r s}$ plus a bounded function, and elementary calculations, that for some suitable constant $\mu_{3}>0$ and almost all $t \in[0, \omega)$,

$$
\left\|\left(z(t)^{T}, \zeta(t)^{T}\right)^{T}\right\|^{r s} \leq \mu_{3}+\mu_{3} \Lambda\left(d_{\lambda}(\theta(\cdot))^{r s}\right)(t)^{r s},
$$

where

$$
\Lambda: \varphi(\cdot) \mapsto\left(t \mapsto \int_{0}^{t} e^{-\tilde{w}_{3}(t-\tau)} \varphi(\tau) d \tau\right)
$$

Using again (3.6), (1.2), (1.6), (1.8), we conclude that the derivative of $d_{\lambda}(\theta)(t)^{2}$ along the solution of (3.3) satisfies, for suitable constants $\mu_{4}, \mu_{5}, \mu_{6}>0, \gamma \in(0, \lambda)$, and almost all $t \in[0, \omega)$,

$$
\begin{aligned}
\frac{\mathrm{d}}{\mathrm{d} t} \frac{1}{2} d_{\gamma}(\theta(t))^{2} & =d_{\gamma}(\theta)\|\theta\|^{-1} \theta^{T} \dot{\theta} \\
& \leq-k d_{\gamma}(\theta)\|\theta\|^{r s}+d_{\gamma}(\theta)\|q(z, \theta+N(\zeta-M \theta))-q(z, 0)+q(z, 0)\| \\
& \leq-k d_{\gamma}(\theta)\|\theta\|^{r s}+d_{\gamma}(\theta)\left[\mu_{q}\left[1+\|\theta+N(\zeta-M \theta)\|^{s}\right]+\mu_{q 0}\left[1+\|z\|^{s}\right]\right] \\
& \leq-k d_{\gamma}(\theta)\|\theta\|^{r s}+\mu_{4} d_{\gamma}(\theta)\left[1+\|\theta\|^{s}+\|N(\eta)\|^{s}+\|z\|^{s}\right] \\
& \leq-k d_{\gamma}(\theta)\|\theta\|^{r s}+\mu_{5} d_{\gamma}(\theta)\left[1+\|\theta\|^{s}+\|\eta\|^{r s}+\|z\|^{r s}\right] \\
& \leq-k d_{\gamma}(\theta)\|\theta\|^{r s}+\mu_{6} d_{\gamma}(\theta)\left[1+\|\theta\|^{r s}+\|\xi\|^{r s}\right] .
\end{aligned}
$$

Now integration of (3.10) over $[0, t) \subset[0, \omega)$, substituting (3.9), invoking Hölder's inequality for $1 / p+1 / q=1$ and $p=r s+1, q=(r s+1) / r s$, using $d_{\gamma}(\theta) \leq d_{\gamma}(\theta) \lambda^{-r s}\|\theta\|^{r s}$ and the fact that $\Lambda$ is a uniformly bounded operator mapping $\mathcal{L}^{k}$-functions into $\mathcal{L}^{k}$-functions, 
we conclude for suitable constants $\mu_{7}, \mu_{8}>0$ and almost all $t \in[0, \omega)$,

$$
\begin{aligned}
\frac{1}{2} d_{\gamma}(\theta(t))^{2}= & \frac{1}{2} d_{\gamma}(\theta(0))^{2}-\int_{0}^{t}\left[k-\mu_{6}\right] d_{\gamma}(\theta)\|\theta\|^{r s} d \tau+\mu_{6} \int_{0}^{t} d_{\gamma}(\theta) d \tau \\
& +\mu_{6} \mu_{3} \int_{0}^{t} d_{\gamma}(\theta)\left[1+\Lambda\left(d_{\lambda}(\theta)^{r s}\right)^{r s}\right] d \tau \\
\leq & \frac{1}{2} d_{\gamma}(\theta(0))^{2}-\int_{0}^{t}\left[k-\mu_{6}\right] d_{\gamma}(\theta)\|\theta\|^{r s} d \tau+\mu_{7}\left\|d_{\gamma}(\theta(\cdot))\right\|_{\mathcal{L}^{1}(0, t)} \\
& +\mu_{7}\|\Lambda\|^{r s}\left\|d_{\gamma}(\theta(\cdot))\right\|_{\mathcal{L}^{p}(0, t)}\left\|d_{\gamma}(\theta(\cdot))^{r s}\right\|_{\mathcal{L}^{q r s}(0, t)}^{r s} \\
\leq & \frac{1}{2} d_{\gamma}(\theta(0))^{2}-\int_{0}^{t}\left[\frac{1}{2} k-\mu_{8}\right] d_{\gamma}(\theta)\|\theta\|^{r s} d \tau-\int_{0}^{t}\left[\frac{1}{2} k-\mu_{8}\right] d_{\gamma}(\theta)^{r s+1} d \tau .
\end{aligned}
$$

Now we proceed in several steps.

STEP 1: We prove $k(\cdot) \in \mathcal{L}^{\infty}(0, \omega ; \mathbb{R})$.

Seeking a contradiction, suppose $k(\cdot)$ is unbounded. Then (3.11) yields $\theta(\cdot) \in \mathcal{L}^{\infty}\left(0, \omega ; \mathbb{R}^{m}\right)$, and hence by $(3.9)(z(\cdot), \zeta(\cdot)) \in \mathcal{L}^{\infty}\left(0, \omega ; \mathbb{R}^{n-1+q}\right)$, so that $(3.10)$ yields $\lim _{t \rightarrow \omega} d_{\gamma}(\theta(t))=$ 0 . Since $\gamma>0$ is arbitrary, this proves $\lim _{t \rightarrow \omega} \theta(t)=0$.

Now (3.5) yields, invoking continuity of $f, g, N, L$ and boundedness of $z(\cdot)$ and $\zeta(\cdot)$, that

$$
\lim _{t \rightarrow \omega}\left\|\delta_{z}(z(t), \zeta(t), \theta(t))\right\|+\left\|\delta_{\zeta}(z(t), \zeta(t), \theta(t))\right\|=0,
$$

and hence, by (3.7), $\lim _{t \rightarrow \omega}(z(t), \zeta(t))=0$. Furthermore, $\lim _{t \rightarrow \omega} \eta(t)=\lim _{t \rightarrow \omega}[\zeta(t)-$ $M \theta(t)]=0$, and therefore, $\lim _{t \rightarrow \omega} y(t)=\lim _{t \rightarrow \omega}[N(\eta(t))+\theta(t)]=0$. By construction of the gain adaptation, we conclude boundedness of $k(\cdot)$.

SteP 2: $\quad$ We prove $(z(\cdot), y(\cdot), \eta(\cdot), \theta(\cdot)),(\dot{z}(\cdot), \dot{y}(\cdot), \dot{\eta}(\cdot), \dot{\theta}(\cdot)) \in \mathcal{L}^{\infty}\left(0, \omega ; \mathbb{R}^{n-1+m+q+m}\right)$. Since $k(\cdot) \in \mathcal{L}^{\infty}(0, \omega ; \mathbb{R})$ is equivalent to $d_{\lambda}(\theta(\cdot)) \in \mathcal{L}^{r s}(0, \omega ; \mathbb{R})$, it is immediate from (3.9) that $(z(\cdot), \zeta(\cdot)) \in \mathcal{L}^{\infty}\left(0, \omega ; \mathbb{R}^{n-1+q}\right)$. To prove that $\theta(\cdot) \in \mathcal{L}^{\infty}\left(0, \omega ; \mathbb{R}^{m}\right)$, rewrite the lower equation in (3.4) as

$$
\dot{\theta}(t)=-\theta(t)+\beta(z(t), \zeta(t), \theta(t))
$$

where, confer (3.10), for suitable constants $\mu_{9}, \mu_{10}>0$ and almost all $t \in[0, \omega)$,

$$
\begin{aligned}
\|\beta(z, \zeta, \theta)\| & \leq\|\theta\|+k\|\theta\|^{r s}+\left\|\delta_{\theta}(z, \zeta, \theta)\right\| \\
& \leq\|\theta\|+k\|\theta\|^{r s}+\mu_{6}\left[1+\|\theta\|^{r s}+\|\zeta\|^{r s}\right] \\
& \leq \mu_{9}+\mu_{9}\left[\|\theta\|^{r s}+d_{\lambda}(\theta)^{r s}-d_{\lambda}(\theta)^{r s}\right] \\
& \leq \mu_{10}+\mu_{10} d_{\lambda}(\theta)^{r s}
\end{aligned}
$$

and hence, since $d_{\gamma}(\theta(\cdot)) \in \mathcal{L}^{r s}(0, \omega ; \mathbb{R})$, it follows from an application of Lemma 2.2 to (3.12) that $\theta(\cdot) \in \mathcal{L}^{\infty}\left(0, \omega ; \mathbb{R}^{m}\right)$.

This also proves that $\eta(\cdot)=\zeta(\cdot)-M \theta(\cdot) \in \mathcal{L}^{\infty}\left(0, \omega ; \mathbb{R}^{q}\right)$, and, by continuity of $N(\cdot)$, $y(\cdot)=N(\eta(\cdot))+\theta(\cdot) \in \mathcal{L}^{\infty}\left(0, \omega ; \mathbb{R}^{m}\right)$.

Finally, boundedness of the derivatives of $z, y, \eta, \theta$ is a consequence of continuous differentiability of $N(\cdot)$ and of (3.2), (3.12).

StEP 3: By Step 1 and 2, standard results of ordinary differential equations yield $\omega=\infty$. Therefore, (i)-(iii) are proved. 
STEP 4: We show (iv):

By Step 3 we have

$$
\frac{\mathrm{d}}{\mathrm{d} t} d_{\lambda_{1}}(y(\cdot))=d_{\lambda_{1}}(y(\cdot))\|y(\cdot)\|^{-1} y(\cdot)^{T} \dot{y}(\cdot) \in \mathcal{L}^{\infty}(0, \infty ; \mathbb{R}),
$$

and since $d_{\lambda_{1}}(y(\cdot)) \in \mathcal{L}^{r_{1}}(0, \infty ; \mathbb{R})$, an application of Barbălat's lemma (see, e.g., [7]) yields the first statement in (iv). The second statement is proved analogously.

STEP 5: We show (v):

Set $\xi^{T}=\left(z^{T}, \zeta^{T}\right)^{T}$ and rewrite the system

$$
\begin{aligned}
\dot{z} & =f(z, \theta+N(\zeta-M \theta)) \\
\dot{\zeta} & =L(\zeta-M \theta)+M q(z, \theta+N(\zeta-M \theta))
\end{aligned}
$$

in the form

$$
\dot{\xi}=\hat{f}(\xi, \theta) .
$$

Since $f(\xi, \theta)$ is locally Lipschitz, there are numbers $c_{1}, c_{2}, m_{1}>0$ such that

$$
\|\hat{f}(\xi, \theta)-\hat{f}(\xi, 0)\| \leq m_{1}\|\theta\|, \quad \text { for all } \quad\|\xi\| \leq c_{1},\|\theta\| \leq c_{2} .
$$

Moreover, the equilibrium $\xi=0$ of $\dot{\xi}=\hat{f}(\xi, 0)$ is by hypothesis locally asymptotically stable, see (1.7), (1.8). Thus, regarding system (3.14) as a system of the form

$$
\dot{\xi}=\hat{f}(\xi, 0)+g(\xi, t),
$$

it follows from [1, pp. 275-6]) that given any $\varepsilon>0$, there exist $\delta_{\varepsilon}>0$ and $\gamma_{\varepsilon}>0$ such that, if $\left\|\xi^{\circ}\right\| \leq \delta_{\varepsilon}$ and $\|\theta(t)\| \leq \gamma_{\varepsilon}$ for all $t \geq 0$, the solution $\xi(t)$ of

$$
\dot{\xi}=\hat{f}(\xi, \theta(t))
$$

satisfying $\xi(0)=\xi^{\circ}$ is such that $\|\xi(t)\| \leq \varepsilon$ for all $t \geq 0$.

We show now that, if $\lambda_{2}$ is small enough, for any $\xi^{\circ}$ there is a time $T=T\left(\xi^{\circ}\right)$ such that the solution $\xi(t)$ of $(3.15)$ satisfying $\xi(0)=\xi^{\circ}$ is such that $\|\xi(T)\| \leq \delta_{\varepsilon}$ and $\|\theta(t)\| \leq \gamma_{\varepsilon}$ for all $t \geq T$. In view of the property recalled above, this proves that $\lim _{t \rightarrow \infty} \max \{0,\|\xi(t)\|-\varepsilon\}=$ 0 . Since $\eta=\zeta-M \theta$ and $\lim _{t \rightarrow \infty} \max \left\{0,\|\theta(t)\|-\lambda_{2}\right\}=0$ (as proven above) this suffices to prove claim $(\mathrm{v})$.

Suppose $\lambda_{2} \leq \gamma_{\varepsilon} / 2$ and observe that, by claim (iv), there is a time $T_{0}$ such that

$$
\|\theta(t)\| \leq 2 \lambda_{2} \leq \gamma_{\varepsilon}, \quad \text { for all } t \geq T_{0} .
$$

Assuming, without loss of generality, $2 \lambda_{2}<1$ we deduce from (3.8) that $\dot{W}(t) \leq-\tilde{w}_{3} W(t)+$ $2 \mu_{2}$, for all $t \in\left[T_{0}, \infty\right)$ and hence

$$
W(t) \leq e^{-\tilde{w}_{3}\left(t-T_{0}\right)} W\left(T_{0}\right)+\frac{2 \mu_{2}}{\tilde{w}_{3}}, \quad \text { for all } \quad t \geq T_{0} .
$$

Substituting (1.8), we conclude the existence of a time $T_{1}>T_{0}$ such that

$$
\|\xi(t)\| \leq R:=\frac{3 \mu_{2}}{w_{1} \tilde{w}_{3}} \quad \text { for all } \quad t \geq T_{1}
$$

Set now

$$
\theta_{T_{1}}(t)= \begin{cases}\theta(t), & \text { if } t \leq T_{1} \\ 0, & \text { if } t>T_{1}\end{cases}
$$


and let $\xi_{T_{1}}(t)$ denote the integral curve of $\dot{\xi}=\hat{f}\left(\xi, \theta_{T_{1}}\right)$ satisfying $\xi_{T_{1}}(0)=\xi^{\circ}$. Indeed, $\xi_{T_{1}}(t)=\xi(t)$ for all $0 \leq t \leq T_{1}$. Moreover, for $t>T_{1}, \xi_{T_{1}}(t)$ is a solution of $\dot{\xi}=\hat{f}(\xi, 0)$ and hence tends to 0 as $t \rightarrow \infty$. In particular, using (1.8), it is seen that there exist numbers $A>0$ and $a>0$ (depending only on $w_{1}, w_{2}, w_{3}$ ) such that

$$
\left\|\xi_{T_{1}}(t)\right\| \leq A e^{-a\left(t-T_{1}\right)}\left\|\xi\left(T_{1}\right)\right\| \quad \text { for all } t \geq T_{1} .
$$

Choosing $T_{2}$ so that $2 A e^{-a T_{2}} R \leq \delta_{\varepsilon}$ yields

$$
\left\|\xi_{T_{1}}\left(T_{1}+T_{2}\right)\right\| \leq \frac{\delta_{\varepsilon}}{2} .
$$

Since $\hat{f}(\xi, \theta)$ is locally Lipschitz, there exists numbers $\ell>0$ and $m_{2}>0$ such that

$$
\left\|\hat{f}(\xi, \theta)-\hat{f}\left(\xi_{T_{1}}, 0\right)\right\| \leq \ell\left\|\xi-\xi_{T_{1}}\right\|+m_{2}\|\theta\|
$$

for all $\|\xi\| \leq R,\left\|\xi_{T_{1}}\right\| \leq A R,\|\theta\| \leq \gamma_{\varepsilon}$.

Let now

$$
\lambda_{2} \leq \min \left\{\frac{\gamma_{\varepsilon}}{2}, \frac{\delta_{\varepsilon} \ell}{4 m_{2} \lambda_{2}\left(e^{L T_{2}}-1\right)}\right\} .
$$

We will show that, at time $T=T_{1}+T_{2}$,

$$
\left\|\xi(T)-\xi_{T_{1}}(T)\right\| \leq \frac{\delta_{\varepsilon}}{2}
$$

which, in view of (3.16), proves that $\|\xi(T)\| \leq \delta_{\varepsilon}$, as requested.

To this end, observe that, for $t \geq T_{1}, \xi(t)$ and $\xi_{T_{1}}(t)$ are integral curves of $\dot{\xi}=\hat{f}(\xi, \theta(t))$ and, respectively, of $\dot{\xi}=\hat{f}(\xi, 0)$ satisfying the same initial condition at time $T_{1}$. Thus, for $t \geq T_{1}$

$$
\xi(t)=\xi\left(T_{1}\right)+\int_{T_{1}}^{t} \hat{f}(\xi(s), \theta(s)) d s
$$

and

$$
\xi_{T_{1}}(t)=\xi\left(T_{1}\right)+\int_{T_{1}}^{t} \hat{f}\left(\xi_{T_{1}}(s), 0\right) d s
$$

Since

$$
\|\xi(s)\| \leq R, \quad\left\|\xi_{T_{1}}(s)\right\|<A R, \quad\|\theta(s)\| \leq 2 \lambda_{2}, \quad \text { for all } s \geq T_{1},
$$

and $2 \lambda_{2} \leq \gamma_{\varepsilon}$, using (3.17), we have

$$
\begin{aligned}
&\left\|\xi(t)-\xi_{T_{1}}(t)\right\| \leq \ell \int_{T_{1}}^{t}\left\|\xi(s)-\xi_{T_{1}}(s)\right\| d s+m_{2} \int_{T_{1}}^{t}\|\theta(s)\| d s \\
& \leq \ell \int_{T_{1}}^{t}\left\|\xi(s)-\xi_{T_{1}}(s)\right\| d s+2 m_{2} \lambda_{2}\left(t-T_{1}\right) .
\end{aligned}
$$

Gronwall's inequality yields

$$
\left\|\xi(t)-\xi_{T_{1}}(t)\right\| \leq \frac{2 m_{2} \lambda_{2}}{\ell}\left(e^{\ell\left(t-T_{1}\right)}-1\right)
$$

and, thus, for $T=T_{1}+T_{2}$,

$$
\left\|\xi(T)-\xi_{T_{1}}(T)\right\| \leq \frac{2 m_{2} \lambda_{2}}{\ell}\left(e^{\ell T_{2}}-1\right) \leq \frac{\delta_{\varepsilon}}{2} .
$$

This completes the proof of claim (v). 


\section{Conclusions}

In [5] it is shown that a certain class of nonlinear systems can be stabilised semiglobally by a dynamic output feedback controller. This is achieved by a high-gain parameter in the feedback law which corresponds to the magnitude of the initial condition. The larger the gain parameter the larger the set of initial conditions so that the closed-loop system stabilises the state. The idea of the present paper is to adapt the gain parameter as a time-varying scalar function depending on the magnitude of $y(t)-N \eta(t)$ where $y(t)$ is the output of the system and $N \eta(t)$ a quantity of the dynamic feedback compensator (see (2.4) for the linear case and (3.1) for the nonlinear case). In the linear case, this adaptive modifications yields global stabilisation and overcomes semiglobal stabilisation as in [5]. In the nonlinear case, we also overcome the semiglobal stabilisation, and even practical stabilisation achieved in [5] is improved by guaranteeing that the output $y(t)$ and the internal variable $\theta(t)$ of the dynamic compensator tends to any prespecified strip $[0, \lambda]$, $\lambda>0$.

\section{References}

[1] Hahn W. (1967): Stability of Motion, Springer-Verlag, Berlin, Heidelberg

[2] Ilchmann A. (1993): Non-Identifier-Based High-Gain Adaptive Control, Springer Verlag, London.

[3] Ilchmann A. (1998): Adaptive $\lambda$-tracking for polynomial minimum phase systems; Dynamics and Stability of Systems 13, 341-371.

[4] Ilchmann A. and Ryan E.P. (1994): Universal $\lambda$-tracking for nonlinearly perturbed systems in the presence of noise, Automatica 30, 337-346.

[5] Isidori A. (1999): Stabilization of nonlinear systems using output feedback, pp. 111133 in: Dynamical Systems, Control, Coding, Computer Vision: new trends, interfaces and interplay, G. Picci, David S. Gilliam (eds.), Birkhäuser, Basel

[6] Isidori A., Teel A. and Praly L. (2000): A note on the problem of semiglobal practical stabilization of uncertain nonlinear systems via dynamic output feedback, Systems and Control Lett., 39, 165-171.

[7] Khalil H.K. (1996): Nonlinear Systems, second ed., Prentice Hall, Upper Saddle River, NJ

[8] Lang S. (1969): Real Analysis, Addison-Wesley, Reading, Massachusetts

[9] Teel A.R. and Praly L. (1995): Tools for semiglobal stabilization by partial state and output feedback. SIAM J. Control Optim. 33, 1443-1485.

[10] Willems J.C. and Byrnes C.I. (1984): Global adaptive stabilization in the absence of information on the sign of the high frequency gain, pp. 49-57 in Lect. Notes in Control and Inf. Sciences 62, Springer-Verlag, Berlin 